Pacific Journal of Mathematics

LAPLACE TRANSFORM METHODS IN MULTIVARIATE

SODA T. TH DORY 


\section{LAPLACE TRANSFORM METHODS IN MULTIVARIATE SPECTRAL THEORY}

ROBERT F. V. ANDERSON

The Laplace transform of the semigroup $\exp (t A)$ generated by an operator $A$ gives the resolvent of $A$. An integral formula is obtained for the Laplace transform of $\exp (t A+B)$, where $B$ is another operator which does not commute with $A$. The new transform has analytic continuation to the same domain as the resolvent, but the analytic continuation is not single-valued. The integral formula is then applied to the joint spectral theory of noncommutative operators. Explicit computations with matrices of degree two illustrate the results.

1. Introduction. Any bounded linear operator $A$ on a Banach space generates a semigroup $\exp (t A), 0 \leqq t<\infty$, and the Laplace transform $\mathscr{L}(s, A)$ of this semigroup converges for Re $s$ sufficiently large and equals the resolvent $(s-A)^{-1} \cdot \mathscr{L}(s, A)$ therefore has unique analytic continuation to the component containing $\infty$ of the resolvent set $A$.

Multivariate problems requiring integration of $\exp \left(\sum t_{i} A_{i}\right)$ one variable at a time, lead us to consider the Laplace transform $\mathscr{L}(s, A, B)$ of $\exp (t A+B), 0 \leqq t<\infty$, where $B$ is a fixed bounded operator.

The main result is:

THEOREM 1. $\mathscr{L}(s, A, B)$ has the contour integral representation (1.1)

$$
(s-A)^{-1}+\int_{s}^{\infty}(u-A)^{-1} B(u-A)^{-1} \exp \left[B(u-A)^{-1}(u-s)\right] d u
$$

valid for $\operatorname{Re} s$ sufficiently large. Therefore, $\mathscr{L}(s, A, B)$ can be analytically continued along any arc not intersecting $\sigma(A)$.

Examples are given in $\$ 5$ which show that the analytic continuation is not always unique.

In $\S \S 3$ and 4 our result is applied to problems in spectral theory. According to the Weyl functional calculus [1], [2], [3], two selfadjoint operators have a joint spectral distribution in the plane, which if $A$ and $B$ commute is simply the tensor product of their spectral measures. Two operators $A$ and $B$ which are merely bounded have instead a two-dimensional Laplace transform $\mathscr{L}(s, \sigma, A, B)$ which if $A$ and $B$ commute is simply the product of their resolvents. $\mathscr{L}(s, \sigma, A, B)$ may be regarded as a functional on the space of entire 
functions on $C^{2}$. Its carrier may be regarded as a joint spectrum of $A, B$. Although there is no unique minimal carrier in general for functionals of this type, Theorem 1 can be exploited to obtain information about the carriers in terms of the spectral properties of $A$ and $B$. It turns out that the actual spectrum of $A$ can be used to construct a carrier, if accuracy with respect to $B$ is sacrificed.

Suppose now that $A$ is bounded and $B$ is self-adjoint. The Weyl calculus for the three self-adjoint operators $\operatorname{Re} A, \operatorname{Im} A$, and $B$ gives a spectrum projecting onto the whole numerical range of $A$. However, in $\S 4$ we construct a hybrid functional for $A$ and $B$ which is an analytic functional with respect to $A$. The motivating question is whether the carrier of this functional will still be the whole numerical range of $A$ or whether the actual spectrum of $A$ will reappear. Theorem 6 gives the transition between the two competing theories and offers no help in shrinking the carrier. But the examples of $\S 5$ show that in some cases the actual spectrum of $A$ does suffice as the carrier.

\section{Proof of Theorem 1 .}

Proof of Theorem 1. The Laplace transform of $\exp (t A+B)$ cannot be computed directly unless $B$ commutes with $A$, in which case the trivial result is:

$$
\mathscr{L}(s, A, B)=\exp (B) \mathscr{L}(s, A) .
$$

We therefore resort to the following contour integral method.

Let $C$ be a simple closed curve containing $\sigma(A)$ (spectrum of $A$ ) in its interior. Then $C$ also encloses $\sigma\left(A+t^{-1} B\right)$ for $|t|$ greater than some constant $k$, and by the Riesz functional calculus [6] applied to the operator $A+t^{-1} B$, for $|t|>k$,

$$
\exp (t A+B)=\exp \left(t\left(A+t^{-1} B\right)\right)=\frac{1}{2 \pi i} \oint_{C} e^{t z}\left[z-\left(A+t^{-1} B\right)\right]^{-1} d z
$$

If in addition

$$
|t|>k_{1}=\sup _{z \notin \operatorname{int} C}\left\|B(z-A)^{-1}\right\|
$$

then

$$
\begin{aligned}
{\left[z-A-t^{-1} B\right]^{-1} } & =\left[\left(I-t^{-1} B(z-A)^{-1}\right)(z-A)\right]^{-1} \\
& =(z-A)^{-1} \sum_{n=0}^{\infty}\left[B(z-A)^{-1}\right]^{n} t^{-n}
\end{aligned}
$$

and 


$$
\begin{aligned}
\exp (t A+B) & =\frac{1}{2 \pi i} \oint_{C} \sum_{n=0}^{\infty} e^{z t} t^{-n}(z-A)^{-1}\left[B(z-A)^{-1}\right]^{n} d z \\
& =\frac{1}{2 \pi i} \oint_{C} \sum_{n, j=0}^{\infty} \frac{z^{j} t^{j-n}}{j !}(z-A)^{-1}\left[B(z-A)^{-1}\right]^{n} d z
\end{aligned}
$$

The double series in the integrand is absolutely and uniformly convergent on the domain $z \in C, \max \left(k, k_{1}\right)<|t|<k_{2}$ where $k_{2}$ is any constant. The contour integral can therefore be evaluated term-byterm. All terms having $j<n$ are $O\left(|z|^{-2}\right)$ for large $z$, and so by enlargement of the contour, they vanish. The remaining terms can therefore be rewritten as the sum

$$
\sum_{q=0}^{\infty} t^{q} \frac{1}{2 \pi i} \int_{C}(z-A)^{-1} \sum_{p=0}^{\infty} \frac{z^{p+q}}{(p+q) !}\left[B(z-A)^{-1}\right]^{p} d z
$$

This power series expansion of the entire function $\exp (t A+B)$ is valid in the annular region given above, and consequently holds for all $t$.

Since $\exp (t A+B)$ has exponential growth rate, the Laplace transform of its power series expansion may be taken term-by-term. This fact is discussed fully in Widder's book on the Laplace transform [8]. Therefore

$$
\mathscr{L}(s, A, B)=\sum_{q=0}^{\infty} \frac{q !}{s^{q+1}} \frac{1}{2 \pi i} \oint_{C}(z-A)^{-1} \sum_{p=0}^{\infty} \frac{z^{p+q}}{(p+q) !}\left[B(z-A)^{-1}\right]^{p} d z .
$$

Next we note that

$$
\sum_{q, p=0}^{\infty} \frac{q !}{s^{q+1}} \frac{z^{p+q}}{(p+q) !}\left[B(z-A)^{-1}\right]^{p}
$$

is absolutely and uniformly convergent when $z \in C,|z / s|<l<1, l$ being any constant less than one.

To reduce the double series to closed form, consider

$$
F(a, b)=\sum_{q, p=0}^{\infty} a^{q} b^{p} \frac{q !}{(p+q) !}
$$

If the series $\sum_{q=0}^{\infty} a^{q+p} q ! /(p+q)$ ! is differentiated $p$ times, we obtain a geometric series which converges to $(1-a)^{-1}$. By elementary means, therefore,

$$
\sum_{q=0}^{\infty} a^{q} \frac{q !}{(p+q) !}=\frac{1}{a^{p}} \int_{0}^{a} \frac{(a-t)^{p-1}}{(p-1) !} \frac{d t}{1-t}, \quad p \geqq 1
$$

and 


$$
\begin{aligned}
F(a, b) & =\frac{1}{1-a}+\sum_{p=1}^{\infty}\left(\frac{b}{a}\right)^{p} \int_{0}^{a} \frac{(a-t)^{p-1}}{(p-1) !} \frac{d t}{1-t} \\
& =\frac{1}{1-a}+b \sum_{p=1}^{\infty} b^{p-1} \int_{0}^{1} \frac{(1-u)^{p-1}}{(p-1) !} \frac{d u}{1-a u} \\
& =\frac{1}{1-a}+b \int_{0}^{1} e^{b(1-u)} \frac{d u}{1-a u} .
\end{aligned}
$$

We now substitute $\alpha=z / s$ and $b=z B(z-A)^{-1}$.

$$
\begin{aligned}
\mathscr{L}(s, A, B) & =\frac{1}{2 \pi i s} \oint_{C}(z-A)^{-1}\left\{\left(1-\frac{z}{s}\right)^{-1}\right. \\
& \left.+z B(z-A)^{-1} \int_{0}^{1} \exp \left[z B(z-A)^{-1}(1-u)\right]\left(1-\frac{z}{s} u\right)^{-1} d u\right\} d z \\
= & (s-A)^{-1}+\int_{0}^{1} \frac{1}{2 \pi i} \oint_{C} z(z-A)^{-1} B(z-A)^{-1} \\
& \cdot \exp \left[z B(z-A)^{-1}(1-u)\right]\left(\frac{s}{u}-z\right)^{-1} d z \frac{d u}{u} .
\end{aligned}
$$

Since the integrand of the contour integral is holomorphic in the neighborhood of $z=\infty$ ontside $C$, the Cauchy integral formula yields

$$
\begin{aligned}
& \mathscr{L}(s, A, B)=(s-A)^{-1} \\
& \quad+\int_{0}^{1} \frac{s}{u}\left(\frac{s}{u}-A\right)^{-1} B\left(\frac{s}{u}-A\right)^{-1} \exp \left[\frac{s}{u} B\left(\frac{s}{u}-A\right)^{-1}(1-u)\right] \frac{d u}{u} .
\end{aligned}
$$

Replacing $u$ by $s / u$, we get Theorem 1 :

$$
\begin{aligned}
\mathscr{L}(s, A, B)= & (s-A)^{-1} \\
& +\int_{s}^{\infty}(u-A)^{-1} B(u-A)^{-1} \exp \left[B(u-A)^{-1}(u-s)\right] d u .
\end{aligned}
$$

Corollary 2. $\mathscr{L}(s, A, B)$ has unique analytic continuation to $R_{\infty}$, the component of the resolvent set of $A$ containing $\infty$, iff for every component $\sigma_{i}$ of $\sigma(A)$ meeting $\bar{R}_{\infty}$, any contour $C_{\imath}$ enclosing component $\sigma_{i}$ only, and for all $j \geqq 1$,

$$
\oint_{C_{i}}(u-A)^{-1}\left[B(u-A)^{-1}\right]^{j} \exp \left[B(u-A)^{-1} u\right] d u=0 .
$$

Proof. Suppose the contour integral of Theorem 1 is continued along two different ares terminating at $s$. The difference between the values of $s$ so obtained is, by homotopy arguments, an integral combination of the closed contour integrals

$$
\oint_{C_{i}}(u-A)^{-1} B(u-A)^{-1} \exp \left[B(u-A)^{-1}(u-s)\right] d u
$$


or

$$
\frac{d}{d s} \oint_{C_{i}}(u-A)^{-1} \exp \left[B(u-A)^{-1}(u-s)\right] d u .
$$

The result follows by power series expansion in $s$.

Corollary 3. The Laplace transform $\mathscr{L}(s, \sigma, A, B)$ of $\exp (t A+$ $\xi B)$ is given by

$$
\sigma^{-1}(s-A)^{-1}+\int_{s}^{\infty}(u-A)^{-1} B(u-A)^{-1}\left[\sigma-B(u-A)^{-1}(u-s)\right]^{-2} d u
$$

for $\sigma>0, s>0$ sufficiently large.

Proof. Replace $B$ by $\xi B$ in the formula for $\mathscr{L}(s, A, B)$. The integration with respect to $\xi$ is elementary.

3. Analytic functionals in spectral theory. Suppose the bounded operators $A_{1}, \cdots, A_{n}$ are all self-adjoint, so that for $\xi \in R^{n}$, $\exp (i \xi \cdot A)$ is a unitary operator. Then by Fourier inversion a tempered distribution $\mathscr{F}^{-1} \exp (i \xi \cdot A)$ is determined. In previous papers by the present author, [1], [2], [3], this distribution was called the "joint spectral distribution" of $A_{1}, \cdots, A_{n}$ and denoted $T(A)$.

In order to gain further insight into this type of spectral distribution, we consider the slightly different case when $i A_{1}, \cdots, i A_{n}$ are assumed only to be the generators of contraction semigroups. This is equivalent to the condition that $A_{1}, \cdots, A_{n}$ have numerical range in the upper half plane. In this case, so does $\xi \cdot A$ if $\xi_{1}, \cdots$, $\xi_{n} \geqq 0$ (abbreviation $\xi \geqq 0$ ), so $\|\exp (i \xi \cdot A)\| \leqq 1$ when $\xi \geqq 0$.

Definition. When $i A_{1}, \cdots, i A_{n}$ generate contraction semigroups, $S(A)$ denotes the tempered distribution defined for $f \in \mathscr{S}\left(R^{n}\right)$ by

$$
S(A) f=(2 \pi)^{-n / 2} \int_{\xi \geq 0}(\mathscr{F} f)(\xi) \exp (i \xi \cdot A) d \xi .
$$

In one dimension, simple computation shows that

$$
S(A) f=\frac{1}{2 \pi i} \int_{-\infty}^{\infty} f(x) \mathscr{L}(x, A) d x
$$

where the Laplace transform $\mathscr{L}(x, A)=(x-A)^{-1}$, provided the spectrum of $A$ does not intersect the real line.

However, $f \in L^{2}\left(R^{1}\right)$ may be written as $f=f_{+}+f_{-}$, where $\mathscr{F} f_{+}=$ $\mathscr{F} f$ for $x \geqq 0, \mathscr{F} f_{-}=\mathscr{F} f$ for $x \leqq 0 . f_{+}$is the boundary value of a function $f_{+}(z)$ holomorphic in the upper half plane, and $\left|f_{+}(z)\right|=$ 
$0\left((\operatorname{Im} z)^{-1}\right)$. If $C$ is any contour in the upper half plane enclosing spectrum $A$, we obtain

$$
S(A) f=\frac{1}{2 \pi i} \oint_{C} f_{+}(z) \mathscr{L}(z, A) d z .
$$

This is just the Riesz calculus (see Ch. XI of [6]), but in two dimensions we can similarly obtain the formula

$$
S(A, B) f=\left(\frac{1}{2 \pi i}\right)^{2} \oint_{C_{1}} \oint_{C_{2}} f_{+}(s, \sigma) \mathscr{L}(s, \sigma, A, B) d s d \sigma
$$

where $\mathscr{L}(s, \sigma)$ is holomorphic for $s, \sigma$ outside $C_{1}, C_{2}$ respectively, and $\mathscr{F} f_{+}=\mathscr{F} f$ for $\xi \geqq 0, \mathscr{F} f_{+}=0$ otherwise.

Formula (3.4) defines a continuous linear functional on the space of entire functions in two complex variables, and the numerical range of $A, B$ need not be restricted. Such functionals are discussed, for example, in Hormanders' book [5]. Such functionals are in one-to-one correspondence with entire functions of exponential growth, in our case $\exp (i \xi \cdot A)$. In one dimension, there is a canonical representation of a functional similar to (3.2), but not in higher dimensions. If $K_{i}$ denotes the compact set bounded by $C_{i}$, and $K=K_{1} \times K_{2}$, then $\|S(A, B) f\| \leqq c \sup _{s, \sigma \in K}|f(s, \sigma)|$, so $K$ is an example of a "carrier" of $S(A, B)$. In general, there is no unique minimal carrier of a functional in dimension greater than 1 .

Lemma 4. If $K_{1}, K_{2}$ contain neighborhoods of the numerical ranges of $A, B$ resp., then $K=K_{1} \times K_{2}$ is a carrier of $S(A, B)$. If $K_{1}$ is simply connected and contains the spectrum of $A$ in its interior, then there exists $K_{2}$ such that $K=K_{1} \times K_{2}$ is a carrier of $S(A, B)$.

Proof. $\mathscr{L}(s, \sigma, A, B)$ is holomorphic when $s, \sigma>0$ if $A$ and $B$ have numerical range in the left half-plane. By translating and rotating $A$ and $B$ independently, the general result is obtained. The second result follows by inspection of formula (2.2) in Corollary 3.

\section{A hybrid functional.}

DEFINITION. Let $i A$ generate a contraction semigroup and let $B$ be self-adjoint. Then the tempered distribution $\mathrm{ST}(A, B)$ is defined for $f \in \mathscr{S}\left(R^{2}\right)$ by

$$
\mathrm{ST}(A, B) f=\frac{1}{2 \pi} \int_{-\infty}^{+\infty} \int_{0}^{\infty}(\mathscr{F} f)(t, \xi) \exp (i(t A+\xi B)) d t d \xi .
$$

Notation. Given $f \in \mathscr{S}\left(R^{2}\right)$, let $f_{+}(z, x)$ denote the analytic con- 
tinuation to $\operatorname{Im} z \geqq 0$ of the function $f_{+} \in L^{2}\left(R^{2}\right)$ satisfying

$$
\begin{array}{cc}
\left(\mathscr{F} f_{+}\right)(t, \xi)=(\mathscr{F} f)(t, \xi), & t \geqq 0 \\
0 & t<0 .
\end{array}
$$

Note that for each fixed $z_{0}, f_{1}\left(z_{0}, x\right) \in \mathscr{S}\left(R^{1}\right)$.

Lemma 5. For each $z$ outside the closure of the numerical range of $A$, there is a tempered distribution $\Phi(z) \in \mathscr{S}^{\prime}\left(R^{1}\right)$ acting on $\varphi(x) \in$ $\mathscr{S}\left(R^{1}\right)$, such that $\Phi(z)$ is (weakly) holomorphic in $z$, and such that for a contour $C$ enclosing the numerical range of $A$,

$$
\operatorname{ST}(A, B) f=\frac{1}{2 \pi i} \oint_{C} \Phi(z) f_{+} d z
$$

Proof. It is easily checked that

$$
\|\exp (t A+i \xi B)\| \leqq \exp (|t|\|A\|) .
$$

Therefore, $\exp (t A+i \xi B)=\sum_{\jmath=0}^{\infty} t^{j} G_{j}(\xi)$ where for all $j, G_{j}(\xi) \in C^{\infty}\left(R^{1}\right)$ and

$$
\left\|G_{j}(\xi)\right\| \leqq\left(\frac{e\|A\|}{j}\right)^{j} \text { uniformly in } \xi .
$$

Therefore, for $\varphi(x) \in \mathscr{S}\left(R^{1}\right)$,

$$
(2 \pi)^{-1 / 2} \int_{-\infty}^{\infty}(\mathscr{F} \varphi)(\xi) \exp (t A+i \xi B) d \xi=\sum_{j=0}^{\infty} t^{j} \Phi_{\jmath}(\varnothing)
$$

where $\Phi_{j}(\varphi)=(2 \pi)^{-1 / 2} \int_{-\infty}^{\infty}(\mathscr{F} \varphi)(\xi) G_{j}(\xi) d \xi$ satisfies

$$
\left\|\Phi_{j}(\varphi)\right\| \leqq(2 \pi)^{-1 / 2}\left(\frac{e\|A\|}{j}\right)^{j}\|(\mathscr{F} \varphi)\| .
$$

In particular, $\Phi_{j}$ is a tempered distribution on $\mathscr{S}\left(R^{1}\right)$. Define

$$
\Phi(z)=\mathscr{L}\left(\sum_{j=0}^{\infty} t^{j} \Phi_{j}\right)=\sum_{j=0}^{\infty} \frac{j !}{z^{j+1}} \Phi_{j}
$$

which converges when $|z|>\|A\|$.

By trivial arguments, $\Phi(z)$ has analytic continuation to all $z$ not in the closure of the numerical range of $A$. The lemma follows immediately for $f$ of the form $\psi(z) \varphi(x)$, which suffices.

TheOREm 6. Suppose $A, B$ act on Hilbert space, and let $A=$ $\operatorname{Re} A+i \operatorname{Im} A$, where $\operatorname{Re} A, \operatorname{Im} A$ are self-adjoint. Then for $\varphi \in \mathscr{S}\left(R^{1}\right)$ and $|z|$ large, 


$$
\Phi(z) \varphi=T(\operatorname{Re} A, \operatorname{Im} A, B) \frac{\varphi\left(x_{3}\right)}{z-\left(x_{i}+i x_{2}\right)}
$$

where $T$ is defined for $g\left(x_{1}, x_{2}, x_{3}\right) \in \mathscr{S}\left(R^{3}\right)$ as stated at the beginning of $\S 3$ and in [1].

Note. The support of the distribution $T$ contains only $\left(x_{1}, x_{2}, x_{3}\right)$ such that $x_{1}+i x_{2}$ is in the closure of the numerical range of $A$. See [1]. Therefore, (4.3) extends at least to all $z$ outside the closed numerical range of $A$.

Proof. Both sides expand in Laurent series in $z$, with coefficient of $z^{-j-1}$ on the left

$$
=(j !) \Phi_{j}(\varphi)=(j !)(2 \pi)^{-1 / 2} \int_{-\infty}^{\infty}(\mathscr{F} \varphi)(\xi) G_{j}(\xi) d \xi
$$

and on the right

$$
\begin{aligned}
& T(\operatorname{Re} A, \operatorname{Im} A, B)\left[\varphi\left(x_{3}\right)\left(x_{i}+i x_{2}\right)^{j}\right] \\
& \quad=(2 \pi)^{-1 / 2} \int_{-\infty}^{+\infty}(\mathscr{F} \varphi)(\xi) T(\operatorname{Re} A, \operatorname{Im} A, B)\left[e^{i x_{3} \xi}\left(x_{i}+i x_{2}\right)^{j}\right] d \xi .
\end{aligned}
$$

Now $G_{j}(\xi)$ is the coefficient of $t^{j}$ in $\exp (t A+i \xi B)$ or $\sum_{n=0}^{\infty} 1 / n !(t A+$ $i \xi B)^{n}$ or, by the monomial substitution rule for $T$ in [1],

$$
\sum_{n=0}^{\infty} \frac{1}{n !} T(\operatorname{Re} A, \operatorname{Im} A, B)\left[e^{t\left(x+i x_{2}\right)} e^{i \xi x_{3}}\right] \text {. }
$$

That is,

$$
G_{j}(\xi)=\frac{1}{j !} T(\operatorname{Re} A, \operatorname{Im} A, B)\left[e^{i x_{3} \xi}\left(x_{i}+i x_{2}\right)^{j}\right] .
$$

Therefore, the two Laurent expansions coincide.

5. Examples. We first examine the hybrid functional in the case when $A$ and $B$ act on the two-dimensional complex Hilbert space.

Let $M_{1}, M_{2}, M_{3}$ be the three hermitian matrices with eigenvalues \pm 1 , satisfying $M_{i} M_{j}+M_{j} M_{i}=0, i \neq j$. (E.g. the Pauli matrices.) Then every $2 \times 2$ complex matrix is a unique linear combination of $M_{1}, M_{2}, M_{3}, I$. Since $I$ commutes with everything, we may as well assume that $A, B$ are linear combinations of $M_{1}, M_{2}, M_{3}$, and up to unitary equivalence and scale changes we can assume $B=M_{1}, A=$ $i \omega \cdot M$ where $\omega$ is a triple of complex numbers.

By simple calculations,

$$
\begin{aligned}
& \exp (A+i \xi B)=I \cos \sqrt{\left(\xi+\omega_{1}\right)^{2}+r^{2}} \\
& +\left(\xi M_{1}+\omega \cdot M\right) \frac{i \sin \sqrt{\left(\xi+\omega_{1}\right)^{2}+r^{2}}}{\sqrt{\left(\xi+\omega_{1}\right)^{2}+r^{2}}}
\end{aligned}
$$


where $r^{2}=\omega_{2}^{2}+\omega_{3}^{2}$. Let $\chi(x)$ denote the characteristic function of the interval $-1 \leqq x \leqq 1$, and let $\delta_{1}, \delta_{-1}$ denote the unit measures concentrated at the points $1,-1$ respectively.

Essentially, the Fourier transforms we need are

$$
\begin{gathered}
\mathscr{F}^{-1} \frac{\sin \sqrt{\xi^{2}+r^{2}}}{\sqrt{\xi^{2}+r^{2}}}=\sqrt{\frac{\pi}{2}} J_{0}\left(r \sqrt{1-x^{2}}\right) \chi(x) . \\
\mathscr{F}^{-1}\left(\cos \sqrt{\xi^{2}+r^{2}}\right)=\sqrt{\frac{\pi}{2}}\left[\delta_{1}+\delta_{-1}+\frac{r J_{1}\left(r \sqrt{1-x^{2}}\right)}{\sqrt{1-x^{2}}} \chi(x)\right] .
\end{gathered}
$$

Since these formulae are rather hard exercises in contour integration, it is worth mentioning that the first is a corollary of the standard formula (see [4]) in dimension 3:

$$
\mathscr{F}^{-1} \frac{\sin |\xi|}{|\xi|}=(2 \pi)^{3 / 2} \mu
$$

where $\mu$ is the uniformly distributed measure on the unit sphere. Our formula follows from this equation by taking the partial Fourier transform with respect to two variables.

In order to obtain $\Phi(z)$, we replace $A$ by $t A$ (i.e., replace $\omega$ by $t \omega)$ and compute the Laplace transform. For the Laplace transforms of the Bessel functions see Watson [7], p. 386. The result is:

Theorem 7. If $B=M_{1}, A=i \omega \cdot M$, then

$$
\begin{aligned}
\Phi(z)= & I \sqrt{\frac{\pi}{2}}\left[\frac{\delta_{1}}{z-i \omega_{1}}+\frac{\delta_{-1}}{z+i \omega_{1}}+\frac{\chi(x)}{\left(\left(z-i \omega_{1} x\right)^{2}+r^{2}\left(1-x^{2}\right)\right)^{3 / 2}}\right] \\
& +\left(-M_{1} \frac{\partial}{\partial x}+i \omega \cdot M \frac{\partial}{\partial z}\right) \sqrt{\frac{\pi}{2}} \frac{\chi(x)}{\left(\left(z-i \omega_{1} x\right)^{2}+r^{2}\left(1-x^{2}\right)\right)^{1 / 2}} .
\end{aligned}
$$

In particular, $\Phi(z)$ can be analytically continued to the complement of the set $\left\{z \mid z=i \omega_{1} x \pm i r \sqrt{1-x^{2}},-1 \leqq x \leqq 1\right\}$ which is the ellipsoid parameterized by $0 \leqq \theta \leqq 2 \pi$,

$$
z=i \omega_{1} \cos \theta+i r \sin \theta .
$$

In the case when $\omega_{2} / \omega_{3}$ is real, this ellipsoid is the boundary of the numerical range of $i A$, and Lemma 5 gives the actual domain of $\Phi(z)$. The other extreme is the case when $\omega_{1}=0$ and $\omega_{2} / \omega_{3}$ is imaginary. Then $\omega_{1}=r=0$ and $\Phi(z)$ is singular only at $z=0$, although the numerical range of $i A$ is the nontrivial ellipsoid $\{z \mid z=$ $\left.i \omega_{2} \cos \theta+i \omega_{3} \sin \theta, 0 \leqq \theta \leqq 2 \pi\right\}$. In the latter case, the singularities of $\Phi(z)$ coincide with the spectrum of 'iA (i.e., $z=0$ ), but the former case shows that the analytic continuation established for $\mathscr{L}(s, A, B)$ does not carry over to the hybrid functional ST $(A, B)$. 
To obtain examples of the Laplace transform, we utilize the elementary fact that when $g \in \mathscr{S}\left(R^{1}\right)$, the Laplace transform is obtained from the Fourier transform by the formula

$$
(\mathscr{L} g)(i s)=\sqrt{2 \pi} \int_{-\infty}^{\infty} \frac{\left(\mathscr{F}^{-1} g\right)(x)}{s-x} d x
$$

One of the coefficients in $\mathscr{L}(s, i B, A)$, with $A, B$ the $2 \times 2$ complex matrices described above, is therefore

$$
\pi \int_{-1}^{1} \frac{J_{0}\left(r \sqrt{1-x^{2}}\right)}{s-x} d x
$$

This coefficient, like the others, has nonunique analytic continuation to all $s \neq \pm 1$. The difference between two values is an integer times $\pi J_{0}\left(r \sqrt{1-s^{2}}\right)$.

This result is easily extended by analytic continuation to any $2 \times 2$ matrices $A, B$.

\section{REFERENCES}

1. R. F. V. Anderson, The Weyl functional calculus, J. Functional Analysis, 4 No. 2, (1969), 240-267.

2. On the Weyl functional calculus, J. Functional Analysis, 6 No. 1, (1970), $110-115$.

3. - The multiplicative Weyl functional calculus, J. Functional Analysis, 9 No. 4, (1972), 423-440.

4. J. Arsac, Fourier Transforms and the Theory of Distributions, Englewood Cliffs, N. J., 1966.

5. L. V. Hormander, An Introduction to Complex Analysis in Several Variables, New York, 1966.

6. F. Riesz and B. Sz-Nagy, Functional Analysis, New York, 1955.

7. G. N. Watson, A Treaties on the Theory of Bessel Functions, 2nd ed., Cambridge University Press, 1944.

8. D. V. Widder, The Laplace Transform, Princeton University Press, 1941.

Received November 19, 1971.

UNIVERSITY OF BRITISH COLUMBia 


\section{PACIFIC JOURNAL OF MATHEMATICS}

\section{EDITORS}

RICHARD ARENS (Managing Editor)

University of California

Los Angeles, California 90024
J. DUGUNDJI*

Department of Mathematics

University of Southern California

Los Angeles, California 90007

D. Gilbarg and J. Milgram

Stanford University

Stanford, California 94305
University of Washington

Seattle, Washington 98105

ASSOCIATE EDITORS
E. F. BECKENBACH
B. H. NeumanN
F. WoLF
K. YosHIDA

\section{SUPPORTING INSTITUTIONS}

\author{
UNIVERSITY OF BRITISH COLUMBIA \\ CALIFORNIA INSTITUTE OF TECHNOLOGY \\ UNIVERSITY OF CALIFORNIA \\ MONTANA STATE UNIVERSITY \\ UNIVERSITY OF NEVADA \\ NEW MEXICO STATE UNIVERSITY \\ OREGON STATE UNIVERSITY \\ UNIVERSITY OF OREGON \\ OSAKA UNIVERSITY
}

\author{
UNIVERSITY OF SOUTHERN CALIFORNIA \\ STANFORD UNIVERSITY \\ UNIVERSITY OF TOKYO \\ UNIVERSITY OF UTAH \\ WASHINGTON STATE UNIVERSITY \\ UNIVERSITY OF WASHINGTON
* * * *
AMERICAN MATHEMATICAL SOCIETY \\ NAVAL WEAPONS CENTER
}

The Supporting Institutions listed above contribute to the cost of publication of this Journal, but they are not owners or publishers and have no responsibility for its content or policies.

Mathematical papers intended for publication in the Pacific Journal of Mathematics should be in typed form or offset-reproduced, (not dittoed), double spaced with large margins. Underline Greek letters in red, German in green, and script in blue. The first paragraph or two must be capable of being used separately as a synopsis of the entire paper. Items of the bibliography should not be cited there unless absolutely necessary, in which case they must be identified by author and Journal, rather than by item number. Manuscripts, in duplicate if possible, may be sent to any one of the four editors. Please classify according to the scheme of Math. Rev. Index to Vol. 39. All other communications to the editors should be addressed to the managing editor, or Elaine Barth, University of California, Los Angeles, California, 90024.

100 reprints are provided free for each article, only if page charges have been substantially paid. Additional copies may be obtained at cost in multiples of 50 .

The Pacific of Journal Mathematics is issued monthly as of January 1966. Regular subscription rate: $\$ 72.00$ a year (6 Vols., 12 issues). Special rate: $\$ 36.00$ a year to individual members of supporting institutions.

Subscriptions, orders for back numbers, and changes of address should be sent to Pacific Journal of Mathematics, 103 Highland Boulevard, Berkeley, California, 94708.

PUBLISHED BY PACIFIC JOURNAL OF MATHEMATICS, A NON-PROFIT CORPORATION

Printed at Kokusai Bunken Insatsusha (International Academic Printing Co., Ltd.), 270, 3-chome Totsuka-cho, Shinjuku-ku, Tokyo 160, Japan.

* C. R. DePrima California Institute of Technology, Pasadena, CA 91109, will replace J. Dugundji until August 1974.

Copyright (C) 1973 by Pacific Journal of Mathematics

Manufactured and first issued in Japan 


\section{Pacific Journal of Mathematics}

\section{Vol. 51, No. $2 \quad$ December, 1974}

Robert F. V. Anderson, Laplace transform methods in multivariate spectral theory .................................................. 339

William George Bade, Two properties of the Sorgenfrey plane . . . . . . . . . . . . 349

John Robert Baxter and Rafael Van Severen Chacon, Functionals on continuous

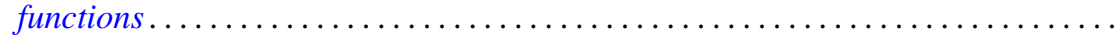

Phillip Wayne Bean, Helly and Radon-type theorems in interval convexity

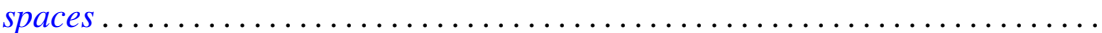

James Robert Boone, On k-quotient mappings $\ldots \ldots \ldots \ldots \ldots \ldots \ldots \ldots \ldots$

Ronald P. Brown, Extended prime spots and quadratic forms . . . . . . . . . . . .

William Hugh Cornish, Crawley's completion of a conditionally upper continuous lattice .............................................

Robert S. Cunningham, On finite left localizations ...................

Robert Jay Daverman, Approximating polyhedra in codimension one spheres

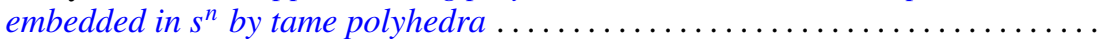

Burton I. Fein, Minimal splitting fields for group representations . . . . . . . . . . . .

Peter Fletcher and Robert Allen McCoy, Conditions under which a connected

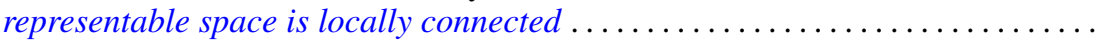

Jonathan Samuel Golan, Topologies on the torsion-theoretic spectrum of a noncommutative ring...

Manfred Gordon and Edward Martin Wilkinson, Determinants of Petrie matrices.

Alfred Peter Hallstrom, A counterexample to a conjecture on an integral condition for determining peak points (counterexample concerning peak points)........

E. R. Heal and Michael Windham, Finitely generated $F$-algebras with applications to Stein manifolds.

Denton Elwood Hewgill, On the eigenvalues of a second order elliptic operator in an unbounded domain ............................

Charles Royal Johnson, The Hadamard product of $A$ and $A^{*}$.

Darrell Conley Kent and Gary Douglas Richardson, Regular completions of Cauchy spaces.

Alan Greenwell Law and Ann L. McKerracher, Sharpened polynomial approximation

Bruce Stephen Lund, Subalgebras of finite codimension in the algebra of analytic functions on a Riemann surface. .

Robert Wilmer Miller, TTF classes and quasi-generators . .

Roberta Mura and Akbar H. Rhemtulla, Solvable groups in which every maximal partial order is isolated ....

Isaac Namioka, Separate continuity and joint continuity...

Alan Saleski, Entropy of self-homeomorphisms of statistical pseudo-metric

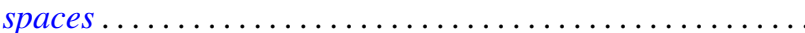

H. A. Seid, Cyclic multiplication operators on $L_{p}$-spaces .....

H. B. Skerry, On matrix maps of entire sequences ............

John Brendan Sullivan, A proof of the finite generation of invariants of a normal

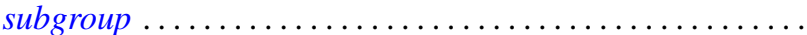

John Griggs Thompson, Nonsolvable finite groups all of whose local subgroups are

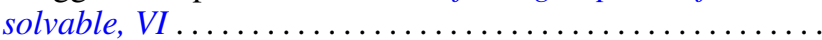

